Cahiers $d u$ MONDE RUSSE

\section{Cahiers du monde russe}

Russie - Empire russe - Union soviétique et États indépendants

$55 / 3-4 \mid 2014$

Varia

\title{
Anne Mézin, Vladislav Rjéoutski, Les Français en Russie au siècle des Lumières, Dictionnaire des Français, Suisses, Wallons et autres francophones en Russie de Pierre le Grand à Paul I ${ }^{\text {er }}$
}

\section{Hélène Rol-Tanguy}

\section{(2) OpenEdition}

\section{Journals}

Édition électronique

URL : http://journals.openedition.org/monderusse/8031

DOI : 10.4000/monderusse.8031

ISSN : $1777-5388$

Éditeur

Éditions de l'EHESS

\section{Édition imprimée}

Date de publication : 1 juillet 2014

Pagination : $362-366$

ISBN : 978-2-7132-2441-6

ISSN : 1252-6576

Référence électronique

Hélène Rol-Tanguy, «Anne Mézin, Vladislav Rjéoutski, Les Français en Russie au siècle des Lumières, Dictionnaire des Français, Suisses, Wallons et autres francophones en Russie de Pierre le Grand à Paul ler », Cahiers du monde russe [En ligne], 55/3-4 | 2014, mis en ligne le 10 avril 2015, Consulté le 25 septembre 2020. URL : http://journals.openedition.org/monderusse/8031 ; DOI : https://doi.org/ 10.4000/monderusse.8031 


\title{
Anne Mézin, Vladislav Rjéoutski, Les Français en Russie au siècle des Lumières, Dictionnaire des Français, Suisses, Wallons et autres francophones en Russie de Pierre le Grand à Paul I ${ }^{\text {er }}$
}

\author{
Hélène Rol-Tanguy
}

\section{RÉFÉRENCE}

Anne MÉzin, Vladislav RjÉoutski, Les Français en Russie au siècle des Lumières, Dictionnaire des Français, Suisses, Wallons et autres francophones en Russie de Pierre le Grand à Paul $\mathrm{I}^{\mathrm{er}}$, Ferney-Voltaire : Publications du Centre international d'étude du XVIII ${ }^{\mathrm{e}}$ siècle, 2011, 2 vol. $1424 \mathrm{p}$.

1 La première mention du Dictionnaire des Français en Russie au xviiie siècle date, à ma connaissance, de $2002^{1}$ et témoigne déjà d'un avancement certain de la recherche. Il aura donc fallu plus de dix ans pour mener à bien ce projet international. Avant cette publication tant attendue, il m'a été possible, grâce à la communication de certains articles par Vladislav Rjéoutski, de mesurer non seulement l'intérêt, mais aussi le caractère indispensable de cet ouvrage pour les chercheurs qui travaillent sur les relations franco-russes de cette époque. Puis, à sa sortie, j'ai pris la mesure du travail réalisé.

2 Les notices biographiques proprement dites se trouvent dans le second volume, alors que le premier, de 580 pages environ, vise à les insérer dans un cadre historique précis. Une trentaine de pages sont consacrées à des informations d'ordre général : la liste des 
collaborateurs, impressionnante par leur nombre, leur diversité et leur qualité ; des cartes de la Russie et des plans des deux capitales. Entre ces deux éléments, prend place un avant-propos d'une dizaine de pages où sont définis les objectifs de l'ouvrage. Suit une introduction substantielle de 140 pages qui retrace l'historique de l'émigration française en Russie tout au long du XviII ${ }^{\mathrm{e}}$ siècle. Le volume est complété par une série d'annexes (dont neuf listes de noms de famille établies de 1734 à 1793 tantôt par les consuls de France, tantôt par les autorités russes), par l'énumération précise des archives consultées en France, en Russie, en Allemagne et en Ukraine, des sources utilisées, manuscrites, imprimées, tant française que russes, et enfin par une riche bibliographie $^{2}$. Il s'achève sur une série d'index qui permettent un maniement très efficace du dictionnaire du second volume. J'y reviendrai, mais attardons-nous d'abord sur l'avant-propos.

Les auteurs n'y négligent pas l'existence de nombreuses études plus ou moins récentes sur les relations franco-russes des origines à nos jours, mais en ce qui concerne la présence française en Russie, c'est "la face visible» qui a été explorée dans des ouvrages généraux sur la diplomatie française ou dans des monographies sur des artistes (architectes, peintres, etc.). Ici, les auteurs poursuivent de nouveaux objectifs : entrer dans le détail de cette présence, faire connaître les expatriés et les voyageurs quels qu'ils soient, célèbres ou anonymes. Or, de ce point de vue, ils constatent que "l'histoire des Français en Russie reste en grande partie à découvrir " ${ }^{3}$ à travers des sources bien souvent inédites.

4 Dans cette optique, l'Introduction s'attache à présenter les différentes étapes qui marquent cette immigration au siècle des Lumières. Le XVIII ${ }^{\mathrm{e}}$ siècle occupe une place cardinale pour cette histoire, car il fut pour la Russie « une période d'apprentissage acharné et d'acquisitions de connaissances» et «les ressortissants de l'Europe occidentale furent ses premiers enseignants ", ce précisément au moment "où l'Europe parlait français $»^{4}$. On remarque alors qu'au fil du siècle, la présence française va se renforcer, non pas tant en nombre qu'en qualité, irriguant la bonne société russe dans de nombreux domaines : enseignement, médecine, commerce, artisanat, construction navale et architecture. L'introduction vise justement à montrer ce cheminement, de Pierre le Grand à Paul Irer.

5 Sous Pierre ${ }^{\text {er }}$, l'émigration française, peu nombreuse, souvent directement recrutée par le tsar avec l'accord des autorités françaises, compte presque exclusivement des ingénieurs et des artisans. Pierre exige d'eux qu'ils forment des Russes dans leurs spécialités respectives, exigence qui sera reconduite sous les règnes suivants : c'est ainsi que le savoir-faire français commence à se répandre en Russie. Mais les conditions de vie consenties à ces expatriés sont telles que, vers 1730, une majorité écrasante des deux cents familles concernées aura quitté l'empire. Quelques grands noms resteront, comme ceux de l'architecte J.-B. Le Blond, le seul à "penser» la ville de Saint-Pétersbourg, et de $\mathrm{Cl}$. Niolon, ingénieur à l'origine de la flotte de guerre russe. Les huguenots, très présents aussi et déjà habitués aux épreuves de l'expatriation, résistent mieux; on les retrouve dans l'armée, la médecine, l'administration civile et l'enseignement.

6 Il faut ensuite attendre la fin du "règne des Allemands" sous Anna Ivanovna et l'accession au pouvoir d'Elisabeth Pétrovna (1741-1762), souveraine francophile, pour voir l'émigration française reprendre. Un état de 1746 recense 206 Français dans la capitale russe: c'est peu, mais désormais la vie pétersbourgeoise "porte le cachet 
culturel [français] qui devait marquer d'une manière indélébile la société russe $»^{5}$. Le nouvel art de vivre de la noblesse russe, né de sa fréquentation des diplomates français, puis des voyages à travers l'Europe, encourage la venue d'artisans spécialisés dans le luxe (bijouterie, coiffure, confiserie, etc.), de secrétaires, de précepteurs et de domestiques, plus ou moins compétents, et enfin d'artistes. Par exemple, dès 1738, un certain Landet y avait fondé une première école de danse et en 1743, une troupe de comédiens français, invités à son initiative, présente à Saint-Pétersbourg des pièces de Molière et de Voltaire. En 1750, le précepteur Feray y ouvre le premier pensionnat français. Par ailleurs, à partir de cette date, des Français et des Suisses cultivés se rendent en Russie, laissent des témoignages écrits sur leur voyage, souvent accompagnés d'éloges à la souveraine, et font connaître le rôle des étrangers, des Français plus particulièrement, dans l'européanisation de la Russie (citons, entre autres, Mainvilliers, Otto Faure, Maugue des Essarts et Fornerod). L'astronome Joseph Nicolas Delisle est la grande figure française de cette époque en Russie, dans le domaine scientifique où les Allemands dominent encore; enseignant l'astronomie à l'Académie des sciences pétersbourgeoise de 1726 à 1747, il y défend les idées de Newton et fonde le département de géographie. Entouré de jeunes Russes, il sera aux origines de la cartographie en Russie.

7 Catherine II (1762-1796) va tenter d'organiser les flux migratoires en promulguant le Manifeste de l'été 1763 où divers avantages sont consentis aux candidats à l'immigration. Elle souhaite peupler les rives sud de la Volga d'agriculteurs, et c'est ainsi que sera créée la communauté des Allemands de la Volga. Mais les Français qui ont répondu à cet appel, n'étant pas agriculteurs pour la plupart, demandent bientôt à résider dans l'une des deux capitales, ce qui leur sera accordé en 1775. C'est donc le gouvernement russe qui est à l'origine de cette nouvelle vague d'immigration française dans ces villes. Cependant, pour l'essentiel, les Français continuent à venir à titre individuel et finissent presque toujours par trouver un emploi.

Après 1789, l'émigration française change de visage, sans être très importante numériquement, car la Russie n'accueille que quelques centaines de personnes. Des membres du clergé, des officiers issus de la grande comme de la petite noblesse y trouvent une situation, les premiers dans l'enseignement, les seconds dans l'armée. Ces derniers, contrairement aux autres émigrés qui se marient à l'intérieur de leur communauté, s'assimileront à la noblesse russe en contractant des mariages qui leur permettent de conserver leur rang.

9 En 1793, il y a ainsi à Saint-Pétersbourg près de 800 Français adultes, dont $20 \%$ sont nés en Russie. Parmi eux, 12 \% font carrière dans l'enseignement, 10 \% font du commerce, $10 \%$ servent dans l'armée. On trouve encore des médecins et des dentistes, des artisans dans les domaines les plus variés et beaucoup de domestiques. L'introduction de l'ouvrage détaillant l'origine géographique de ces émigrés, la Lorraine, le Dauphiné, ou encore des villes comme Paris ou Lyon, met en évidence le phénomène bien connu de «l'émigration en chaîne ".

10 Les artistes sont nombreux, comme l'architecte Vallin de la Mothe, responsable de la construction de plusieurs bâtiments à Saint-Pétersbourg, et l'on compte toujours des peintres, des comédiens, des musiciens. À la fin du siècle, la scène musicale pétersbourgeoise s'affirme comme l'une des plus brillantes d'Europe. Cette grande variété des professions exercées révèle une intervention permanente des Français dans la vie de la bonne société russe, dans son quotidien (les grandes maisons de commerce 
tenues par des Français se développent à partir des années 1780), comme dans le domaine culturel.

11 Nous émettrons pourtant quelques réserves sur cette dernière partie si bien documentée. Les auteurs qui annonçaient au départ leur choix d'une présentation chronologique de l'émigration y dérogent en introduisant un nouvel axe : l'étude de la présence française par professions. Suivre tout au long du siècle l'évolution de la place occupée par les médecins, les précepteurs, les commerçants français face à leurs concurrents étrangers ou locaux présente un indiscutable intérêt. Ainsi on découvre les détails de la réussite du commerce français à Moscou dans l'édition musicale, la fabrication des cartes à jouer ou dans la librairie, avec l'organisation de salles de lecture et le prêt de livres. Mais pour avoir un tableau d'ensemble de cette question, le lecteur est amené à faire des retours en arrière puisque des informations de cet ordre qui se trouvent déjà dans les périodes précédentes sont complétées là. Par ailleurs on appréciera le sous-chapitre dédié aux formalités que sont tenus d'accomplir les Français pour entrer en Russie et s'y installer tandis qu'un autre chapitre, intitulé "Réseaux français», nous renseigne sur leur vie quotidienne, leurs liens avec leur communauté comme avec la population locale et leur degré d'assimilation.

Le second volume (837 pages) offre des notices concernant environ 7000 personnes. Les unes ne comptent que quelques lignes, avec les maigres renseignements biographiques que les auteurs ont pu glaner sur le personnage, ses dates de séjour en Russie et son activité professionnelle si elle est connue: d'Arnaud Masson, par exemple, sont mentionnées sa profession d'origine, tapissier de haute lisse aux Gobelins, ses dates d'arrivée en 1717 et de départ en 1723 ; Nicolas Massé est un Marseillais devenu maître d'hôtel de Catherine II. Pour d'autres, figure le parcours de toute une famille : ainsi, les Chapuzeau, apparentés au calviniste français Samuel Chapuzeau et venus de Hanovre, dont la carrière militaire est suivie sur trois générations. Les ambassadeurs de France et le personnel de l'ambassade font l'objet de notices développées - citons celle consacrée au chevalier Charrette de La Colinière qui retrace très précisément le travail de ce chargé d'affaires placé auprès du comte de Ségur de 1784 à 1786. Enfin des notices concernent des personnalités incontournables, comme Falconet - l'histoire de la statue équestre de Pierre le Grand est rapportée -, avec tous ses mécomptes -, ou La Harpe, "gouverneur général » du futur Alexandre $\mathrm{I}^{\mathrm{er}}$; les deux hommes resteront en relation jusqu'à la mort du souverain. Et il y a la notice sur Diderot; Georges Dulac y met particulièrement en évidence la présence éminente de la Russie dans l'élaboration de la pensée du philosophe. À la fin de chaque notice sont données les références des sources utilisées.

Les différents index contenus dans le premier volume ouvrent de nouvelles possibilités d'exploration. À côté du Répertoire socioprofessionnel et de l'Index des lieux de naissance ou de provenance, c'est l'Index général qui reprend non seulement les entrées du second volume, mais beaucoup des noms cités dans les notices mêmes. Le mélange des noms propres, des toponymes et des thèmes abordés surprend au début, mais il est sans incidence sur la recherche. Par ailleurs l'accès aux noms des Russes qui sont, pour une raison ou pour une autre, entrés en relation avec des Français, permet de corriger des noms malmenés dans une correspondance ou de découvrir celui de personnes qualifiées jusque-là par leur seule profession. Ainsi à l'entrée «Stroganov, Alexandre Sergueïevitch ", on apprend le nom de son valet de chambre, Charles Frères, et l'on a un accès immédiat aux noms des compositeurs, des peintres, des littérateurs et 
des diplomates français qui l'ont fréquenté (même si l'on s'étonne de l'absence du chevalier de Corberon).

On aura compris qu'en dépit de quelques réserves, l'ouvrage d'Anne Mézin et de Vladislav Rjéoutski marque une étape majeure dans l'étude de la présence française en Russie au XVIII ${ }^{\mathrm{e}}$ siècle et, comme le souhaitent ses auteurs, il sera un instrument " pour aller plus loin » dans la recherche. Ils suggèrent d'ailleurs quelques pistes à la fin de l'introduction du premier volume. La publication de nouvelles archives amènera sans doute la découverte de nouveaux noms et de compléments biographiques en faveur de ceux déjà présents dans ce dictionnaire qui, comme j'ai pu le vérifier lors de certaines rencontres, suscite intérêt et envie chez les spécialistes des autres langues ${ }^{6}$.

\section{NOTES}

1. Anne Mézin, Vladislav Rjéoutski, «Projet d'un dictionnaire des Français en Russie au XVIII ${ }^{\mathrm{e}}$ siècle », Cahiers du Monde russe, $n^{\circ} 43$ (2-3), 2002, p. 343-354.

2. Plus loin, après les annexes, on appréciera la présence d'une chronologie allant de la naissance de Pierre $\mathrm{I}^{\mathrm{er}}$ à l'assassinat de Paul $\mathrm{I}^{\mathrm{er}}$, de la Table des rangs, ainsi que d'un tableau des poids et mesures, et de précisions sur les toponymes, les rangs et les institutions sans doute particulièrement bienvenus pour les non-spécialistes de la Russie

3. Anne Mézin, Vladislav Rjéoutski, Les Français en Russie au siècle des Lumières..., 2011, p. 4.

4. Ibidem, p. 3.

5. Ibidem, p. 4.

6. En 2014, l'Institut Pierre le Grand de Saint-Pétersbourg se propose de publier en russe la partie du dictionnaire qui concerne l'ensemble du règne de ce souverain. L'introduction et les notices seront donc enrichies à cette occasion de matériaux relatifs au début du règne.

\section{AUTEURS}

\section{HÉLĖNE ROL-TANGUY}

Centre d'Histoire « Espaces et cultures» (CHEC), Université Blaise-Pascal - Clermont II 